\title{
Envelhecimento bem-sucedido e vulnerabilidade em saúde: aproximações e perspectivas
}

Henrique Salmazo da Silva ${ }^{1}$

Ângela Maria Machado de Lima²

Ricardo Galhardoni ${ }^{3}$

SILVA, H.S.; LIMA, A.M.M.; GALHARDONI, R. Successful aging and health vulnerability: approaches and perspectives. Interface - Comunic., Saude, Educ., v.14, n.35, p.867-77, out./dez. 2010.

Considering the multidimensional and dynamic nature of the concept of health vulnerability, this article aimed to promote a discussion about the meaning of aging successfully even in the presence of vulnerabilities. We have selected studies which discussed dimensions of vulnerability in the context of old age, exploring personal, social, programmatic and community resources that could make older adults be less vulnerable in situations of dependence and reduced functional capacity. The articulation of the concept of health vulnerability and successful and healthy aging seems to be productive and should be further explored.

Keywords: Health. Vulnerability. Elderly.
Tomando como referência a natureza multidimensional e dinâmica do conceito de vulnerabilidade em saúde, o objetivo deste artigo foi promover uma discussão sobre o que vem a ser envelhecer de forma bem-sucedida mesmo na presença de vulnerabilidades. Foram selecionados estudos que discutiram dimensões da vulnerabilidade no contexto da velhice, problematizando os recursos pessoais, sociais, programáticos e comunitários que poderiam tornar as pessoas idosas menos vulneráveis em face das situações de dependência e capacidade funcional reduzida. Embora pouco aplicada no campo da Gerontologia, a articulação entre o conceito de vulnerabilidade em saúde e o envelhecimento saudável e bem-sucedido parece ser produtiva e precisa ser melhor explorada.

Palavras-chave: Saúde. Vulnerabilidade. Idoso.

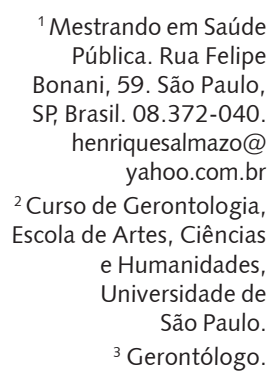




\section{Introdução}

A possibilidade de se tomar o envelhecimento como processo positivo e a velhice como etapa da vida que pode ser acrescida de bem-estar, prazer e qualidade de vida vem sendo objeto de pesquisadores e estudiosos interessados em compreender as condições associadas à saúde na última fase do ciclo de vida: a velhice. Nesta discussão, trataremos a velhice como um fenômeno social e vital complexo, sendo determinado pelo curso de vida e pelas experiências e oportunidades socioculturais e históricas acumuladas durante a vida (Lima, Silva, Galhardoni, 2008; Paz, Santos, Eidt, 2006; Glass, 2003).

Ancorando-se pela visão de que é possível manter o bem-estar e a qualidade de vida na velhice, os conceitos de envelhecimento ativo e bem-sucedido têm levantado discussões sobre o envelhecimento saudável, enfatizando que a velhice e o envelhecimento não são sinônimos de doença, inatividade e contração do desenvolvimento (Lima, Silva, Galhardoni, 2008; Neri, 2008). Contudo, não existe consenso na literatura a respeito da saúde na velhice. A vulnerabilidade em saúde é um conceito pouco estudado na Gerontologia e que poderá trazer contribuições interessantes para enriquecer essa discussão.

Em estudo de revisão, Paz, Santos e Eidt (2006) enumeraram as condições sociais e de saúde que, empiricamente, podem estar envolvidas no contexto da vulnerabilidade em saúde no processo de envelhecimento, entre elas: a capacidade funcional na velhice, a distribuição das doenças crônico-degenerativas, a disponibilidade de programas e serviços, a posição social que os indivíduos ocupam e os recursos sociais disponíveis.

Apesar de esses autores apresentarem a relação entre a vulnerabilidade e a saúde, atualmente, a terminologia "vulnerabilidade" tem sido utilizada para se referir aos idosos com susceptibilidade para desenvolver incapacidades ${ }^{4}$, ou para indicar os idosos com condições sociais desfavoráveis e que possuiriam menos acesso a oportunidades de atingir níveis satisfatórios de saúde e independência. A noção de "risco", associada ao desenvolvimento de condições desfavoráveis de saúde, aparece subjacente nessas aplicações. Contudo, nem todos os idosos com susceptibilidade a incapacidades as desenvolvem, e nem todos os idosos com condições sociais desfavoráveis possuirão piores condições de saúde (Hildon et al., 2010). Acreditamos que a interação entre saúde, independência e autonomia na velhice solicita um olhar analítico e que pode receber contribuições do conceito de vulnerabilidade em saúde.

Oriundo das Ciências Humanas e da Saúde, o conceito propõe que as condições populacionais de saúde ultrapassam as correlações de risco, sugerindo que o estado de saúde é intrínseco aos sujeitos e ao coletivo (Muñoz Sánchez, Bertolozzi, 2007). O conceito possui raiz vinculada aos Direitos Humanos e começa a ser construído na década de 1980 para se compreender a distribuição dos casos de HIV (vírus da imunodeficiência adquirida) em populações de homossexuais norte-americanos.

A ampla divulgação de práticas e programas de divulgação contra o HIV contribuiu para a diminuição de casos entre os homossexuais, tornando limitadas as concepções de risco e fatores de risco naquele contexto. O conceito é concebido considerando-se, fundamentalmente, três aspectos: o plano individual, o plano social e o plano programático e de serviços (Muñoz Sánchez, Bertolozzi, 2007; Ayres et al., 2003).

De acordo com Ayres et al. (2003), o plano da vulnerabilidade pessoal referese a comportamentos que os indivíduos adotam que podem ser favoráveis ou desfavoráveis para o autocuidado e o estado de saúde. Já o plano da

${ }^{4}$ Termo que indica deficiências e limitações no desempenho das atividades e restrições na participação social. 
vulnerabilidade social enfatiza que as representações sociais, os estigmas e as condições sociais podem ser fatores que contribuem para o status de saúde, valorizando também o acesso aos meios de comunicação e a disponibilidade de usufruir de recursos cognitivos e materiais. Já o plano da vulnerabilidade programática e de serviços sugere que o acesso, a qualidade dos programas e o compromisso das instituições são determinantes importantes para a distribuição das condições de saúde e bem-estar.

Cabe destacar que a vulnerabilidade em saúde é um conceito apropriado para estimular respostas sociais e que não possui aplicação direta às questões que envolvem a saúde na velhice. Nesse sentido, pode-se indagar se o idoso é socialmente reconhecido como um cidadão de direitos e que, independentemente da condição social, possui o direito de alcançar o envelhecimento saudável e apresentar uma velhice bem-sucedida (Brasil, 2003).

$\mathrm{Na}$ Gerontologia, o conceito de velhice bem-sucedida, inicialmente, foi proposto por Havighurst no início da década de 1960, sugerindo que envelhecer bem era produto da participação em atividades sendo essas associadas à satisfação, manutenção da saúde e participação social. A proposição desse conceito foi uma mudança ideológica importante no estudo do envelhecimento, incentivando a investigação sobre os aspectos positivos na velhice e sobre o potencial de desenvolvimento associado ao envelhecimento (Neri, 2008; Rowe, Kahn, 1998). Ao final da década de 1980 e início da década de 1990, o conceito foi resignificado e passou a ser tratado por dois modelos teóricos: o de Rowe e Kahn (1998), que foi baseado nos dados do estudo norte-americano "MacArthur Foundation Study", e o de Baltes e Baltes (1990), que propôs o modelo teórico de "Seleção, Otimização e Compensação (SOC)" para representar, no âmbito das ciências psicológicas, as possibilidades de plasticidade comportamental e funcional em face das perdas associadas às últimas fases do ciclo de vida.

O modelo proposto por Rowe e Kahn (1998) sugere que o envelhecimento bem-sucedido seria composto por três fatores: engajamento com a vida; manutenção de altos níveis de habilidades funcionais e cognitivas e baixa probabilidade de doença; e incapacidade relacionada à prática de hábitos saudáveis para redução de riscos. Embora esses fatores sejam importantes, esse modelo possui limitações por tratar como secundárias as dimensões socioculturais e coletivas associadas à saúde na velhice e por poucos idosos se enquadrarem nesse modelo (Britton et al., 2008).

Baltes e Baltes (1990) propuseram que os idosos que conseguem usar mecanismos de compensação e regulação das perdas associadas ao envelhecimento são aqueles que alcançam a velhice bem-sucedida e atingem níveis satisfatórios de independência. Ao propor o modelo SOC, os autores sugerem que os idosos selecionam as tarefas que acreditam possuírem melhor ou ótimo desempenho, excluindo ou evitando aquelas com as quais possuem dificuldades; aperfeiçoam as habilidades que ainda estão preservadas, utilizando esforços para mantê-las; e compensam as habilidades comprometidas, como é o caso das estratégias mnemônicas para manter a eficiência da aquisição e resgate de novas informações.

Em face das limitações conceituais do modelo proposto por Rowe e Kahn (1998), Kahn (2003) incentiva que o debate sobre envelhecimento bem-sucedido seja acrescido pelo modelo teórico proposto por Baltes e Baltes (1990) e por investigações que consideram a autopercepção dos idosos sobre o estado de saúde.

Além dos modelos destacados, a política de envelhecimento ativo, proposta pela Organização Mundial de Saúde (OMS, 2005), também tem discutido as questões relacionadas à saúde na velhice, enfatizando que envelhecer bem faz parte de uma construção coletiva e que deve ser facilitado pelas políticas públicas e por oportunidades de acesso à saúde ao longo do curso de vida. Desse modo, a definição de envelhecimento ativo baseia-se na "otimização das oportunidades de saúde, participação, segurança, com o objetivo de melhorar a qualidade de vida à medida que as pessoas ficam mais velhas" (OMS, 2005, p.13). A priori, a política parte do pressuposto de que, para se envelhecer de forma saudável e bem-sucedida, é preciso favorecer oportunidades para que os indivíduos possam optar por estilos de vida saudáveis e, ainda, fazer controle do próprio status de saúde.

Tomando como referência a natureza multidimensional e dinâmica do conceito de vulnerabilidade, o objetivo deste artigo é contribuir para a reflexão sobre a saúde na velhice e problematizar questões importantes no cenário do envelhecimento no Brasil (Alexandre, Cordeiro, Ramos, 2009; Ramos, 2007; Santos, Lopes, Neri, 2007; Camarano, 2006; Ramos, 2003). Para tanto, foram selecionados estudos que 
discutiram dimensões da vulnerabilidade no contexto da velhice e que se concentram nas seguintes questões:

a) Refletir o que vem a ser envelhecer de forma satisfatória ou bem-sucedida, mesmo na presença de vulnerabilidades. Isso é possível? Em que medida, pensando que os idosos brasileiros possuem baixo nível de escolarização e de renda, e dificuldades no uso e acesso aos serviços de saúde?

b) Problematizar que as situações de dependência não implicam, necessariamente, condições de vulnerabilidade, isto é, alguns idosos dependentes e com diferentes graus de limitação funcional dispõem de recursos pessoais, sociais e comunitários que poderiam torná-los menos vulneráveis em saúde e, consequentemente, menos expostos a determinadas condições desfavoráveis de saúdedoença. Imagina-se que esta última perspectiva auxiliará a identificar os pontos que precisarão de intervenções e os recursos que os idosos possuem para manejar as situações de dependência.

Espera-se que o presente artigo possa contribuir para a formulação de estudos e práticas no campo da Gerontologia, iniciando, assim, discussões acerca do que vem a ser envelhecer de forma bemsucedida, mesmo na presença de vulnerabilidades.

\section{Vulnerabilidade programática: \\ qual a contribuição das práticas e serviços para o bem-estar na velhice?}

Pensar na vulnerabilidade programática associada ao envelhecimento bem-sucedido não é um exercício fácil e, muito menos, trivial. É preciso considerar que, nas últimas décadas, o envelhecimento populacional brasileiro aconteceu de forma mais acelerada do que em países europeus, acarretando escassez de programas de suporte e de contingência às necessidades sociais e de saúde da população idosa. Há que se entender, como cita Neri (2005), que o Brasil não atingiu o "walfare state" e não o atingirá tão prontamente, pois, com as desigualdades sociais, econômicas, educacionais, o idoso foi pouco privilegiado. Apesar do Estatuto do Idoso (2003) e da Política Nacional do Idoso (1994), muitas vezes, outras faixas de idade acabam por concorrer com os idosos pelos mesmos recursos.

Dessa forma, olhar para a trajetória de concepção da lei que promulga, respectivamente, o Estatuto do Idoso e a Política Nacional do Idoso nos permite ter uma interpretação mais sólida do que ocorre no cenário nacional em relação às ações programáticas empreendidas pelo Estado para combater as vulnerabilidades dos idosos. Partindo da premissa de que a velhice é uma categoria socialmente construída, Groisman (1999) aponta, como marco inicial dessa categoria no Brasil, o ano de 1890, quando surge, no Rio de Janeiro, o asilo São Luiz para a velhice desamparada e, em 1909, um pavilhão para velhos não-desamparados.

Borges (2002) atenta para a dificuldade de se traduzirem políticas pensadas para os idosos em ganhos reais. No Brasil, não há estudos longitudinais que se ocupem das questões que envolvem os conceitos de envelhecimento bem-sucedido e vulnerabilidade; além do campo acadêmico, há que se ressaltar que os programas de atenção em Gerontologia são amplos e possuem múltiplas aplicações. Apesar da heterogeneidade entre o perfil dos programas e do público atendido, as práticas e os serviços direcionados de atenção ao idoso possuem, como princípio, algumas diretrizes legais regulamentadas ao longo das décadas de 1990 e 2000. São direcionamentos da Política Nacional do Idoso, do Estatuto do Idoso e da RDC 2005: a valorização da autonomia, do bem-estar, socialização e independência da pessoa idosa.

Clark et al. (2008) defendem a hipótese de que, além do formato dos programas, as práticas de atenção ao idoso são fundamentais para o sucesso das intervenções. Esta afirmação se baseia nos resultados que os autores levantaram ao entrevistarem uma população de idosos norte-americanos com renda domiciliar inferior a $200 \%$ da média e um grupo populacional de idosos com maior renda. Ao compará-los em relação aos hábitos e crenças sobre a saúde, esses autores encontraram que: o grupo de idosos com menor renda referiu a medicação e o cuidado direto de saúde como os principais meios de autogerenciar a saúde, enquanto os idosos com melhor renda exploraram dimensões como atividade física e engajamento em atividades mentais; as relações com família se baseavam entre trocas de apoio para os idosos de baixa renda e entre visitas para os idosos de alta renda, encontrando-se maior presença de conflitos familiares entre o primeiro grupo; a diferença entre os grupos também se baseou nas expectativas 
que os idosos possuíam em relação ao futuro, à saúde e à possibilidade de terem um envelhecimento bem-sucedido. Os idosos de baixa renda referiram que já haviam vivido bastante e citavam poucos projetos para o futuro, enquanto os de alta renda declararam que havia muitos projetos pessoais.

Em outras palavras, os dados de Clark et al. (2008) sugerem que as práticas valorizem a percepção dos idosos em relação ao autogerencimento do cuidado em saúde, visto que pode ser uma ferramenta importante para a eficácia das intervenções e para a acessibilidade de populações em desvantagem social.

Lima (2003) examinou as relações entre autocuidado e envelhecimento nas narrativas de idosos residentes em regiões empobrecidas do município de São Paulo. A autora partiu da premissa de que assistimos a modos heterogêneos de conceber, de experimentar e de gerenciar o envelhecimento. Apontou que a gestão da velhice, durante muito tempo considerada como própria da vida privada e familiar, nos meados do século XX, ganhou expressão e legitimidade no campo das preocupações sociais e transformou-se em uma questão da esfera pública. Porém, recentemente, o avanço das idades sofreu um processo de reprivatização, que recoloca o envelhecer e seus destinos sobre a responsabilidade individual, abrindo espaço, então, para que a velhice seja relativizada no leque das preocupações sociais do momento (Debert, 1999).

Nessa perspectiva, o autocuidado não pode ser compreendido como simples adoção de saberes técnicos para a promoção da saúde, mas como uma atitude prática, relacionada à experiência de envelhecer, às condições de vida e às interações familiares e comunitárias. Trata-se da necessidade de resistir à tendência de responsabilização individual do idoso pela sua saúde, frequentemente associada às propostas de autocuidado.

No âmbito da eficácia das intervenções e programas gerontológicos, Huss et al. (2008) conduziram um estudo de meta-análise que avaliou o impacto das visitas domiciliares sobre indicadores como institucionalização, declínio funcional e mortalidade. Os autores incluíram vinte estudos de segmento, publicados em bases de dados, como PUBMED e MEDLINE, entre os anos de 2001 e 2007. Apesar da heterogeneidade dos dados e do desenho metodológico, os resultados indicaram que houve associação estatisticamente significativa entre visitas domiciliares e preservação ou aumento do status funcional entre os dados que incluíram avaliações clínicas $(p=0.02)$. Já para o indicador mortalidade, as visitas parecem ter sido mais protetoras para os idosos jovens (risco relativo $=0,74$ ) do que entre os idosos mais longevos (risco relativo $=1,14$ ). Para as medidas institucionalização e tipos de intervenções, não foram encontradas associações estatísticas, fator que pode ter sido atribuído à heterogeneidade dos dados. Este estudo revela o papel protetor de programas de visita e atenção domiciliar, sobretudo entre os idosos mais jovens, provavelmente com melhor estado de saúde e com maior probabilidade de se beneficiarem da intervenção ao longo do tempo.

Mellis et al. (2008) conduziram um estudo randômico para comparar o custo e efetividade de um programa de atenção multidisciplinar e de um programa convencional, ambos direcionados para idosos frágeis e vivendo em comunidade. Adotou-se, no estudo, o critério de Fried et al. (2001), em que o fenótipo de fragilidade engloba: perda de peso não intencional (10\% do peso inicial ao mês); relato de fadiga e exaustão; diminuição da força de preensão; redução das atividades físicas; e diminuição da velocidade para executar a marcha. Os custos foram calculados pelos cuidados prestados e os recursos materiais e humanos envolvidos; já a efetividade foi avaliada por meio do desempenho funcional e cognitivo, com intervalo de seis meses entre as avaliações.

Os resultados levantados pelos autores indicaram que, dos 151 idosos reavaliados, 34 foram tratados de forma bem-sucedida, demonstrando aumento de cerca de dez pontos nas medidas de desempenho funcional e estabilidade de desempenho cognitivo. Desses 34, 27 pertenciam ao grupo de idosos que receberam atenção multiprofissional, e o estudo revelou que a atenção multidisciplinar propiciou redução dos gastos para hospitalização, institucionalização e cuidados de enfermagem; embora tenha solicitado aumento dos gastos para cuidados adicionais, home-care, cuidados diários e refeições recebidas em casa. A estimativa de custo-efetividade indicou que, para se obterem $95 \%$ de chances de sucesso no tratamento, seria necessário investir 34 mil euros.

Os investimentos do governo em programas de auxílio e renda também parecem ter uma influência positiva sobre as medidas de saúde na velhice. Neri e Soares (2007), por meio dos dados da PNAD - 
Pesquisa Nacional por Amostragem de Domicílios, estimaram o impacto das políticas públicas de transferência de renda sobre a autoavaliação de saúde e sobre o acesso aos serviços de atenção em saúde, comparando os dados das populações entrevistadas em 1998 e em 2003. Os dados analisados pelos autores indicaram que a proporção de idosos que autoavaliaram a saúde como boa ou muito boa subiu de $36,5 \%$ em 1998 para 40,8\% em 2003. Entre os idosos com idade superior a 65 anos e com renda domiciliar de meio salário-mínimo, verificou-se diminuição do percentual de idosos que estiveram acamados de 1998 a 2003.

Em face dos dados apresentados, as intervenções de saúde podem considerar o contexto em que os idosos se inserem e o compartilhamento de decisões na proposição de ações que almejam promover o bem-estar e a manutenção da independência do idoso (Lima, Silva, Galhardoni, 2008). Nessa perspectiva, usar a senecultura (momento em que o indivíduo se prepara para o envelhecimento e a sociedade se prepara para o envelhecimento do indivíduo) seria fundamental para: consolidar programas baseados na prevenção, promoção da saúde, mobilização comunitária, formação de profissionais sensíveis às questões do envelhecimento e ampliação do acesso a informações sobre saúde e qualidade de vida. Esses elementos permitiriam minimizar as situações de vulnerabilidade social e vulnerabilidade programática, tornando as intervenções qualificadas e compromissadas com as condições de vida dos idosos atendidos (Jacob-Filho, 2006; Moon, 2002).

É importante lembrar que, como cita Sposati (2001), é preciso desenvolver uma política que favoreça não só as pessoas com vulnerabilidade social, mas toda a população, fazendo com que todos tenham as mesmas oportunidades de acesso. Cabe ao Estado pensar na efetivação dos princípios propagados na Constituição de 1988 a respeito da integralidade em saúde, fugindo das falsas premissas de que: 1) a velhice sempre está diretamente associada à doença; 2) que o envelhecimento é um processo que pressupõe homogeneidade; 3 ) que os idosos são sujeitos passivos das intervenções propostas pelos serviços e rede de atenção social e de saúde, não dispondo de recursos pessoais e sociais que poderiam influenciar o resultado das intervenções; e 4) que fatores intrínsecos e extrínsecos, associados ao gênero e classe social, não mudam o peso da "equação" e que isso não muda diretamente os padrões de envelhecimento saudável e patológico (Neri, 2005).

\section{Vulnerabilidade individual: existem recursos pessoais que poderiam favorecer $a$ independência?}

Os estudos que se concentraram em investigar o impacto de recursos pessoais ${ }^{5}$ sobre o estado de saúde e capacidade funcional consideraram, fundamentalmente, dois níveis de análise: os idosos saudáveis e os idosos que possuem valores intermediários de capacidade funcional (Kempen et al., 2006; Seeman, Chen, 2003).

Kempen et al. (2006), ao estudarem idosos saudáveis, investigaram a presença de fatores de proteção ${ }^{6}$ e de risco sobre a trajetória da funcionalidade na velhice. Os autores avaliaram 1.765 idosos no intervalo de oito anos (entre 1993 e 2001). Os resultados revelaram que, entre o grupo com maior declínio funcional, foi observada maior projeção de doenças crônico-degenerativas, altos níveis de neuroticismo e maior incidência de sintomas depressivos e avaliação negativa da saúde. Ajustando-se os dados segundo idade, gênero e anos de estudo, os autores encontraram que a autopercepção positiva da saúde e elevados níveis de autoeficácia ${ }^{7}$ foram fatores de proteção para o desempenho funcional, enquanto os sintomas depressivos e a presença de baixo senso de domínio foram fatores

\author{
5 Termo empregado, \\ nesta discussão, como \\ recursos que são \\ inerentes ao indivíduo e \\ que englobam \\ autopercepção, \\ comportamentos, \\ relações interpessoais, \\ satisfação pessoal e bem- \\ estar. \\ ${ }^{6}$ Termo empregado, em \\ estudos epidemiológicos, \\ como um fator de \\ exposição que minimiza \\ as chances de os \\ indivíduos apresentarem \\ o evento observado \\ nesse caso, a diminuição \\ da funcionalidade. \\ ${ }^{7}$ Avaliação individual de \\ que as ações pessoais \\ fazem a diferença nas \\ relações interpessoais e \\ nos aspectos de \\ funcionamento da vida \\ diária.
}


associados a menores níveis de desempenho. Estes dados revelam que somente evitar os fatores de risco como medida preventiva limita a análise de fatores de proteção, apresentando-se como substancialmente diferentes.

Seeman e Chen (2003) analisaram a relação entre variáveis psicossociais e de estilo de vida sobre o nível de funcionalidade entre idosos com doenças crônico-degenerativas - entre elas: hipertensão, diabetes, câncer, doenças cardiovasculares e fraturas - e idosos com ausência de doenças crônicodegenerativas. Os autores encontraram que, na avaliação de segmento (2,5 anos), o indicador "ter pouco conflito com os outros" foi preditivo de menor declínio funcional entre os idosos com diabetes e depressão; em idosos com câncer, o nível de autoeficácia (crenças nas próprias habilidades) se associou com estabilidade de desempenho; e os idosos com doenças cardiovasculares tiveram menor declínio quando reportaram maior suporte emocional. Em face dos resultados apresentados, os autores sugeriram que os fatores sociais e psicológicos foram críticos para a funcionalidade em idosos com doenças crônico-degenerativas.

Outra linha de investigação se concentra em mapear recursos internos e externos orientados para adaptação e enfrentamento aos estressores e às condições de saúde, o que poderia tornar os idosos com limitações funcionais menos susceptíveis a determinados eventos de saúde-doença (Bastistone, Fortes, Yassuda, 2007; Neri, 2007; Piazza, Charles, Almeida, 2007; Néri, Fortes, 2006; Kirby, Coleman, Daley, 2004; Poon et al., 2003). Produto das ciências psicológicas, o estudo do senso de ajustamento psicológico propõe que, mesmo na presença de baixos níveis de saúde física e níveis de funcionalidade reduzidos, os idosos podem apresentar níveis satisfatórios de qualidade de vida e bem-estar subjetivo, 0 que, potencialmente, atenuaria a velocidade de progressão do declínio funcional (Park-Lee et al., 2009; Bowling et al., 2007; Néri, 2007; Ostir, Ottenbacher, Markides, 2004; Pennix, Guralnik, BandeenRoche, 2000).

Pennix, Guralnik e Bandeen-Roche (2000) investigaram o impacto da vitalidade emocional em 1.002 idosas com idade superior a 65 anos e com desempenho limitado para duas ou mais Atividades de Vida Diária (AVDs). Os autores definiram vitalidade emocional como um constructo que envolveu senso de domínio pessoal, senso de felicidade e baixos níveis de depressão e ansiedade. Os resultados encontrados revelaram que 351 mulheres idosas possuíam vitalidade emocional, sendo que a vitalidade emocional esteve significativamente associada à preservação das AVDs e à manutenção do desempenho funcional.

Alguns estudos, como os de Ostir, Ottenbacher e Markides (2004) e Park-Lee et al. (2009), investigaram o impacto dos afetos positivos sobre a incidência do fenótipo de fragilidade proposto por Fried et al. (2001). Ostir, Ottenbacher e Markides (2004) acompanharam 1.558 idosos não frágeis durante o intervalo de sete anos e encontraram incidência de fragilidade em 7,9\% do total dos idosos pesquisados. No estudo, os afetos positivos, definidos como estados de contentamento e felicidade, diminuíram o risco de os idosos apresentarem os critérios do fenótipo de fragilidade na avaliação final. Park-Lee et al. (2009), na mesma direção, estudaram a relação entre os afetos positivos e a incidência de fragilidade em 3.167 idosos cuidadores e 617 não cuidadores, avaliados no intervalo de dois anos. A projeção de afetos positivos e a incidência de fragilidade foram similares em ambos os grupos, todavia, o grupo de idosos cuidadores obteve maiores níveis de estresse percebido.

No estudo de Bowling et al. (2007), em uma amostra de 999 idosos ingleses, cerca de 31\% apresentavam declínio significativo nas habilidades funcionais. Destes, $62 \%$ referiam a própria saúde como boa. Dentre outros fatores, foram preditivos para a autoavaliação positiva de saúde: possuir maior percepção de controle sobre a vida, o envolvimento social e possuir maior rede de contatos sociais.

Em outras palavras, as evidências apresentadas sugerem que o estudo do bem-estar, saúde e qualidade de vida na velhice também poderia considerar como os idosos, mesmo na presença de baixo desempenho funcional, adaptam-se e utilizam estratégias pessoais e/ou recursos internos e externos frente aos baixos níveis de desempenho. A articulação dessas questões com as intervenções gerontólogicas precisa ser melhor elucidada, estabelecendo a relação com a vulnerabilidade social e o impacto das condições sociais na trajetória de saúde ao longo do curso de vida. 


\section{Considerações finais}

Em geral, a contribuição dos estudos populacionais e conduzidos com a população idosa centra-se na possibilidade de se mapear objetivamente o perfil de saúde dos idosos, fornecendo dados importantes sobre o impacto das condições sociais para as medidas objetivas e subjetivas de saúde. Apesar das contribuições, as estimativas de risco são limitadas quando se ampliam para o plano individual e subjetivo dos indivíduos (Ayres et al., 2003).

Cabe destacar aqui os limites do conceito de vulnerabilidade e o desafio de articulá-lo com dimensões da saúde na velhice. Assim como descrevem Ayres et al. (2003), ao utilizarmos o conceito, corremos o risco de vitimizarmos os indivíduos e de não produzirmos respostas sociais que produzam o efeito desejado (ampliação paralisante). Ao se identificarem grupos populacionais, pode-se tutelar ou vitimizar os indivíduos, propiciando discriminações negativas. No mesmo sentido, o efeito paralisante do conceito pode advir da amplitude e complexidade para transformar as situações de vulnerabilidade em respostas sociais, traduzindo-as para o plano das políticas públicas e das intervenções (Ayres et al., 2003).

$\mathrm{Na}$ discussão levantada, a articulação com as questões relativas ao processo de envelhecimento poderá exigir respostas sociais que nem sempre serão atingidas; e ainda podem propiciar a discriminação de idosos com maiores ou menores níveis de vulnerabilidade. Como menciona Debert (1999), é preciso ampliar a concepção socialmente vigente de que os idosos são os únicos responsáveis pela condição de saúde que atingem na velhice. A discriminação, advinda da adoção de hábitos de vida não favoráveis ao longo do curso de vida, precisa ser desmistificada, enfatizando-se a necessidade de se oferecerem oportunidades iguais para o acesso à saúde.

Ressaltamos aqui que procuramos desenvolver uma primeira tentativa de aproximar o conceito de vulnerabilidade em saúde à saúde na velhice, apresentando limitações que se relacionam ao alcance dos estudos descritos e por não ter abrangido toda a complexidade do fenômeno.

No contexto de populações com vulnerabilidade social, os estudos poderão traçar metas para o enfrentamento dos determinantes sociais e para a solução dos problemas levantados pelos próprios idosos. Nesse sentido, problematizar temas que envolvem o cotidiano dos idosos com vulnerabilidade social, programática e/ou individual, poderia resultar na valorização do papel do idoso como sujeito de direitos, e, da comunidade, como corresponsável para a promoção do envelhecimento bem-sucedido. Como indica Clark et al. (2008), é preciso oferecer condições para que os idosos, em especial os idosos de baixa renda, possam autogerenciar a saúde e ter outras perspectivas em relação aos significados de envelhecimento bem-sucedido e das estratégias para alcançá-lo.

Em síntese, a articulação do conceito de vulnerabilidade às questões levantadas parece ser produtiva, apesar de ainda pouco explorada na literatura. As propostas de práticas à luz do conceito de vulnerabilidade, sugeridas neste trabalho, são: estratégias que os próprios idosos utilizam para alcançar a saúde e o bem-estar; os recursos individuais e intrínsecos aos idosos e que poderiam ser utilizados como meios de favorecerem as condições de saúde; a disponibilidade e a adequação de recursos e serviços direcionados à população idosa, em especial os idosos com baixa renda e com algum tipo de limitação funcional; as respostas sociais incentivadas e fomentadas pelos idosos.

Para tanto, é fundamental se oferecerem condições estruturais para que as respostas sociais às situações de vulnerabilidade ocorram, envolvendo: profissionais, a sensibilização do poder público, organizações e movimentos sociais, cuidados comunitários de longa duração, empresas e profissionais que atuam na capacitação e formação (Moon, 2002). São necessários esforços coletivos que possam minimizar as situações de vulnerabilidade da população idosa brasileira, favorecendo, assim, o alcance do envelhecimento saudável e com qualidade de vida. 


\section{Colaboradores}

Henrique Salmazo da Silva, Ângela Maria Machado de Lima e Ricardo Galhardoni trabalharam juntos em todas as etapas de produção do manuscrito.

\section{Referências}

ALEXANDRE, T.S.; CORDEIRO, R.C.; RAMOS, L.R. Fatores associados à qualidade de vida de idosos ativos. Rev. Saude Publica, v.43, n.4, p.613-21, 2009.

AYRES, J.R.C.M. et al. O conceito de vulnerabilidade e as práticas de saúde: novas perspectivas e desafios. In: CZERESNIA, D.; FREITAS, C.M. (Orgs.). Promoção da saúde: conceitos, reflexões, tendências. Rio de Janeiro: Fiocruz, 2003. p.117-40.

BALTES, P.B.; BALTES, M.M. Successful aging: perspective from the behavioral sciences. Cambridge: Cambridge University Press, 1990.

BATISTONE, S.S.T.; FORTES, A.C.G.; YASSUDA, M.S. Aspectos psicológicos do envelhecimento. In: FORLENZA, O.V. (Org.). Psiquiatria geriátrica: do diagnóstico precoce à reabilitação. São Paulo: Atheneu, 2007. p.32-7.

BORGES, M.C.M. Gestão participativa em organizações de idosos: instrumentos para a promoção da cidadania. In: FREITAS, E.L. et al. (Eds.). Tratado de geriatria e gerontologia. Rio de Janeiro: Guanabara Koogan, 2002. p.1037-41.

BOWLING, A. et al. Quality of life among older people with poor functioning: the influence of perceived control over life. Age Ageing, v.36, n.3, p.310-5, 2007.

BRASIL. Ministério da Saúde. Estatuto do idoso. 1.ed. 2.reimpr. Brasília: Ministério da Saúde, 2003.

BRITTON, A. et al. Successful aging: the contribution of early-life and midlife risk factors. J. Am. Geriatr. Soc., v.56, n.6, p.1098-105, 2008.

CAMARANO, A.A. Envelhecimento da população brasileira: uma contribuição demográfica. In: FREITAS, E.L. et al. (Eds.). Tratado de geriatria e gerontologia. 2.ed. Rio de Janeiro: Guanabara Koogan, 2006. p.88-105.

CLARK, D.O. et al. The meaning and significance of self-manegement among socioeconomically vulnerable older adults. J. Gerontol. B Psychol. Sci. Soc. Sci., v.63B, n.5, p.5312-8, 2008.

DEBERT, G.G. A reinvenção da velhice: sociabilização e processos de reprivatização do envelhecimento. São Paulo: Editora da Universidade de São Paulo, 1999.

FRIED, L. et al. Frailty in older adults: evidence for a phenotype. J. Gerontol. A Biol. Sci. Med. Sci., v.56, n.3, p.146-56, 2001.

GLASS, T. Successful aging. In: TALLIS, R.C.; FILLIT, H.M. (Eds.). Brocklehurt's textbook of geriatric medicine and gerontology. 6.ed. Nova York: Churchill Livingstone, 2003. p.173-99.

GROISSMAN, D. A infância do asilo: a institucionalização da velhice no Rio de Janeiro da virada do século. 1999. Dissertação (Mestrado) - Instituto de Medicina Social, Universidade Federal do Rio de Janeiro, Rio de Janeiro. 1999.

HILDON, Z. et al. Examining resilience of life in the face of health-related and psychosocial adversity at older ages: what is "right" about the way we age? Gerontologist, v.50, n.1, p.36-47, 2010.

HUSS, A. et al. Multidimensional preventive home visit programs for communitydwelling older adults: a systematic review and meta-analysis of randomized controlled trials. J. Gerontol. A Biol. Sci. Med. Sci., v.63, n.3, p.298-307, 2008. 
JACOB-FILHO, W. Velhice fragilizada: aspectos genéticos e ambientais e conceitos sobre fragilização da vida na quarta idade - abordagem médica. In: SEMINÁRIO DE VELHICE FRAGILIZADA, 2006, São Paulo. Comunicação oral... São Paulo: SESC, 2006.

KAHN, R.L. Successful aging: intended and unintended consequences of a concept. In: POON, L.W.; GUELDNER, S.H.; SPROUSE, B.M. (Eds.). Successful aging and adaptation with chronic diseases. Nova York: Springer Publishing Company, 2003. p.55-82.

KEMPEN, G.I.J.M. et al. Risk and protective factors of different functional trajectories in older persons: are these the same? J. Gerontol. B Psychol. Sci. Soc. Sci., v.61, n.2, p.95-101, 2006.

KIRBY, S.E.; COLEMAN, P.G.; DALEY, D. Spirituality and well-being in frail and nonfrail older adults. J. Gerontol. B Psychol. Sci. Soc. Sci., v.59, n.3, p.123-9, 2004.

LIMA, A.M.M. Saúde no envelhecimento: o autocuidado como questão. 2003. Tese (Doutorado) - Faculdade de Medicina, Universidade de São Paulo, São Paulo. 2003.

LIMA, A.M.M.; SILVA, H.S.; GALHARDONI, R. Envelhecimento bem-sucedido: trajetórias de um constructo e novas fronteiras. Interface - Comunic., Saude, Educ.., v.12, n.27, p.795-807, 2008.

LIMA-COSTA, M.F. et al. Desigualdade social e saúde entre idosos brasileiros: um estudo baseado na Pesquisa Nacional por Amostra de Domicílios. Cad. Saude Publica, v.19, n.3, p.745-57, 2003.

MELLIS, R.J.F. et al. Cost-effectiveness of a multidisciplinary intervention model for community-dwelling frail older people. J. Gerontol. A Biol. Sci. Med. Sci., v.63, n.3, p.275-82, 2008.

MOON, O-R. Alternative systems of health and welfare service delivery for older persons in developed and developing countries. In: WORLD HEALTH ORGANIZATION. Development of health and welfare systems: adjusting to ageing. Valencia: WHO, 2002. p.19-40.

MUÑOZ SÁNCHEZ, A.I.; BERTOLOZZI, M.R. Pode o conceito de vulnerabilidade apoiar a construção do conhecimento em Saúde Coletiva? Cienc. Saude Colet., v.12, n.2, p.319-24, 2007.

NERI, A.L. Palavras-chave em gerontologia. Campinas: Alinea, 2008.

Qualidade de vida na velhice e subjetividade. In: (Org.). Qualidade de vida na velhice: enfoque multidisciplinar. Campinas: Alínea, 2007. p.13-59.

As políticas de atendimento aos direitos da pessoa idosa expressas no estatuto do idoso. A Terceira Idade, v.16, n.34, p.7-24, 2005.

NERI, A.L.; FORTES, A.C.G. A dinâmica do estresse e enfrentamento na velhice e sua expressão no prestar cuidados a idosos no contexto da família. In: FREITAS, E.L. et al. (Eds.). Tratado de geriatria e gerontologia. 2.ed. Rio de Janeiro: Guanabara Koogan, 2006. p.1277-88.

NERI, M.C.; LOPES, W.S. Estimando o impacto da renda na saúde através de programas de transferência de renda aos idosos de baixa renda no Brasil. Cad. Saude Publica, v.23, n.8, p.1845-56, 2007.

ORGANIZAÇÃO MUNDIAL DA SAÚDE - OMS. Envelhecimento ativo: uma política de saúde. Brasília: Organização Pan-Americana de Saúde, 2005.

OSTIR, G.V.; OTTENBACHER, K.J.; MARKIDES, K.S. Onset of frailty in older adults and the protective role of positive affect. Psychol. Aging, v.19, n.3, p.402-8, 2004. 
PARK-LEE, E. et al. Positive affect and incidence of frailty in elderly women caregivers and noncaregivers: results of caregiver-study of osteoporotic fractures. J. Am. Geriatr. Soc., v.57, n.4, p.627-33, 2009.

PAZ, A.A.; SANTOS, B.R.L.; EIDT, O.R. Vulnerabilidade e envelhecimento no contexto da saúde. Acta Paul. Enferm., v.19, n.3, p.338-42, 2006.

PENNIX, B.W.J.H.; GURALNIK, J.M.; BANDEEN-ROCHE, K. The protective effect of emotional vitality on adverse health outcomes in disabled older women. J. Am.

Geriatr. Soc., v.48, n.11, p.1359-66, 2000.

PIAZZA, J.R.; CHARLES, S.T.; ALMEIDA, D.M. Living with chronic health conditions: age differences in affective well-being. J. Gerontol. B Psychol. Sci. Soc. Sci., v.62, n.6, p.313-21, 2007.

POON, L.W. et al. Coping with comorbidity. In: POON, L.W.; GUELDNER, S.H.; SPROUSE, B.M. (Eds.). Successful aging and adaptation with chronic diseases. Nova York: Springer Publishing Company, 2003. p.116-50.

RAMOS, L.R. Fatores determinantes do envelhecimento saudável em idosos residentes em centro urbano: projeto Epidoso, São Paulo. Cad. Saude Publica, v.19, n.3, p.793-8, 2003.

RAMOS, M. Impact of socioeconomic status on Brazilian elderly health. Rev. Saude Publica, v.41, n.4, p.616-24, 2007.

ROWE, J.W.; KAHN, R. Successful aging. New York: Pantheon Books, 1998.

SANTOS, G.A.; LOPES, A.; NERI, A.L. Escolaridade, raça e etnia: elementos de exclusão social de idosos. In: NERI, A.L. (Org.). Idosos no Brasil: vivências, desafios e expectativas na terceira idade. São Paulo: Perseu Abramo/Edições SESC SP, 2007. p.6579.

SEEMAN, T.; CHEN, X. Risk and protective factors for physical functioning in older adults with and without chronic conditions: the MacArthur study of sucessfull aging. In: POON, L.W.; GUELDNER, S.H.; SPROUSE, B.M. (Eds.). Successful aging and adaptation with chronic diseases. Nova York: Springer Publishing Company, 2003. p.83-103.

SPOSATI, A. Desafios para fazer avançar a política de assistência social no Brasil. Rev. Serv. Soc., v.22, n.68, p.55-82, 2001.

SILVA, H.S.; LIMA, A.M.M.; GALHARDONI, R. Envejecimiento feliz y vulnerabilidad en salud: aproximaciones y perspectivas. Interface - Comunic., Saude, Educ., v.14, n.35, p.867-77, out./dez. 2010.

Tomando como referencia la naturaleza multi-dimensional y dinámica del concepto de vulnerabilidad en salud, el objetivo de este artículo es el promover una discusión sobre lo que viene a ser envejecer con éxito incluso en presencia de vulnerabilidades. Se han seleccionado estudios que discuten dimensiones de vulnerabilidad en el contexto de la vejez, encarando la problemática de los recursos personales, sociales, programáticos y comunitarios que podrían tornar a las personas ancianas menos vulnerables ante situaciones de dependencia y capacidad funcional reducida. Aunque poco aplicada en el campo de la Gerontología, la articulación del concepto de vulnerabilidad en salud y el envejecimiento saludable y feliz parece ser productiva y ha de ser mejor utilizada.

Palabras clave: Salud. Vulnerabilidad. Anciano. 